\title{
Tratamiento de heridas complejas mediante fibrina rica en plaquetas y leucocitos (L-PRF), experiencia inicial de un centro
}

\author{
Ignacio Andrés Araya C. ${ }^{1}$, Cristopher Mayer O. ${ }^{1}$, Francisco Javier Pinedo H. ${ }^{1}$ y \\ Julio Antonio Jiménez L. ${ }^{1}$
}

\section{Treatment of complex wounds using platelet rich fibrin and leukocytes (L-PRF), initial experience of a center}

Introduction: The use of platelet concentrates for the treatment of complex wounds and tissue regeneration is being widely used worldwide. During the last time, the second generation of platelet concentrates, particularly L-PRF, has made it possible to effectively treat patients with this pathology. Due to its low cost and versatility, it has been possible to apply this technique in various clinical situations with good results. The objective of this work is to present our experience using L-PRF for the healing of complex wounds (HCC) as an alternative to the use of grafts of different degrees of complexity. Materials and Method: A prospective analysis was carried out with a series of cases who underwent surgical treatment of complex wounds using L-PRF at Santiago Oriente - Luis Tisné Brousse Hospital, between the months of January 2017 and December 2018. Through clinical examination and previously defined inclusion, success, and failure parameters, a total of 11 patients with complex wounds were evaluated who underwent local treatment with an L-PRF graft. Results: The etiology of the wounds was varied. $8(72 \%)$ of the cases achieved $100 \%$ epithelialization and $3(28 \%)$ failed. Predisposing factors for the failure of the technique were identified, and it was also possible to establish a predictive relationship of success where a high probability of epithelialization is related when the granulation of the wound occurs during the first 10 days on the L-PRF graft. Conclusion: The treatment of complex wounds using L-PRF is a feasible alternative, with low cost and requirements (compared to the use of grafts, flaps and dermal substitutes) and safe in the resolution of complex wounds, allowing to reduce morbidity, the costs associated with treatment and hospital stay. Key words: complex wounds; plasma rich in fibrin and leukocytes; L-PRF.

\section{Resumen}

Introducción: El uso de concentrados plaquetarios para el tratamiento de heridas complejas y regeneración tisular está siendo ampliamente utilizado a nivel mundial. Durante el último tiempo, la segunda generación de concentrados plaquetarios, particularmente el L-PRF, ha permitido tratar de manera efectiva a pacientes con esta patología. Debido a su bajo costo y versatilidad, ha sido posible aplicar esta técnica en variadas situaciones clínicas con buenos resultados. El objetivo de este trabajo es presentar nuestra experiencia utilizando L-PRF para la curación de heridas complejas (CHC) como una alternativa al uso de injertos de distinto grado de complejidad. Materiales y Método: Se realizó un análisis prospectivo de una serie de casos de pacientes que fueron sometidos a tratamiento quirúrgico de heridas complejas mediante el uso de L-PRF en el Hospital Santiago Oriente - Luis Tisné Brousse, entre los meses de enero de 2017 y diciembre de 2018. Mediante examen clínico y parámetros de inclusión, de éxito y de fracaso definidos previamente, se evaluó un total de 11 pacientes con heridas complejas a los cuales se les realizó un tratamiento local con injerto de L-PRF. Resultados:_La etiología de las heridas fue variada. 8 (72\%) de los casos lograron una epitelización del 100\% y 3 (28\%) fracasaron. Se identificaron factores predisponentes para el fracaso de la técnica, y también fue posible establecer una relación de predicción de éxito en donde se relaciona una probabilidad alta de epitelización cuando la granulación de la herida ocurre durante los primeros 10 días sobre el injerto de L-PRF. Conclusión: El tratamiento de heridas complejas mediante L-PRF es una alternativa factible, de bajo costo y requerimientos (comparada con el uso de injertos, colgajos y sustitutos dérmicos), es segura en la resolución de heridas complejas, permitiendo disminuir la morbilidad, los costos asociados al tratamiento y estadía hospitalaria.

Palabras clave: heridas complejas; plasma rico en fibrina y leucocitos; L-PRF.
${ }^{1}$ Hospital Santiago Oriente Dr. Luis Tisné. Universidad de Chile. Santiago, Chile.

Recibido 2020-06-02 aceptado 2020-10-05

Correspondencia a: Dr. Ignacio Andrés Araya C. ignacioaraya@uchile.cl 


\section{Introducción}

La utilización de productos derivados de la sangre para su uso complementario en la reparación de heridas, controlar el sangrado y estimular su curación se inició en el período de la segunda guerra mundial, con el uso combinado de trombina y fibrinógeno ${ }^{1,2}$. La técnica se perfeccionó posteriormente con los pegamentos o colas de fibrina, las que constituyen un concentrado de fibrinógeno obtenido a través de la polimerización inducida por trombina y calcio ${ }^{3}$. Esto fue descrito por primera vez en 1970 por Matras $\mathrm{H}$, quien continúo sus estudios y publicó su utilidad clínica en $1982^{4}$. Por otro lado, otros investigadores en la década de los ochenta ampliaron sus usos a otras áreas como la cirugía plástica, la cirugía de cabeza y cuello, la cirugía oral y maxilofacial, la traumatología y la neurocirugía.

La elaboración de colas de fibrina es un proceso complejo y costoso, por lo que se han descrito diferentes protocolos que han permitido una elaboración menos compleja. Es así como surgieron los concentrados plaquetarios, utilizados principalmente para potenciar la cicatrización, tal como fue descrito inicialmente por Whitman et al. ${ }^{5}$, con un gel de plaquetas para su aplicación en el campo de la cirugía maxilofacial.

Existen diferentes técnicas de obtención de concentrados plaquetarios. Una de las más conocidas corresponde a la primera generación de éstos, el plasma rico en plaquetas (PRP). La segunda generación, el plasma rico en fibrina (PRF), fue desarrollado por Choukroun ${ }^{6}$ inicialmente en el área de la periodoncia. Se ha visto un gran potencial de esta técnica en la reparación de heridas mucosas y cutáneas, con la ventaja de ser una técnica simple obtenida mediante el centrifugado de la sangre completa sin aditivos anticoagulantes.

El L-PRF o plasma rico en fibrina y leucocitos es un concentrado plaquetario que, además, tiene incorporado gran cantidad de leucocitos en el coágulo de fibrina, a diferencia del PRF convencional. La técnica de obtención del L-PRF es sencilla y de bajo costo, mediante la extracción de sangre periférica venosa o arterial. La sangre se transfiere directamente a tubos de plástico de 6 a $9 \mathrm{ml}$ sin ningún aditivo ni anticoagulante e inmediatamente se centrifuga a $2.700 \mathrm{rpm}$ durante 12 minutos ( $\approx 400 \mathrm{~g}$ ) a temperatura ambiente ${ }^{7,8}$. El volumen de sangre obtenido se calibra según el área a cubrir de la herida, siendo aproximadamente de $72 \mathrm{cc}$ a $144 \mathrm{cc}$ por sesión.

Los concentrados plaquetarios contienen elevadas cantidades de factores de crecimientos claves en la reparación de heridas como la proteína morfogénica ósea 2 (BMP2), el factor de crecimiento derivado de plaquetas (PDGF-AB), el factor de crecimiento transformador beta 1 (TGF $\beta-1)$, el factor de crecimiento insulínico tipo 1 (IGF), el factor de crecimiento epidérmico (EGF) y el factor de crecimiento endotelial vascular (VEGF). Estos tienen la capacidad de estimular la remodelación de la matriz extracelular, potenciar la angiogénesis, estimular la proliferación y migración celular?. Se ha demostrado que estos factores de crecimiento en el L-PRF presentan una liberación continua de al menos 7 días ${ }^{8,9}$.

El uso clínico del L-PRF, ha sido probado con múltiples propósitos: tratamiento de heridas complejas $^{7}$, úlceras corneales ${ }^{11}$, gastrectomías ${ }^{12}$, perforaciones timpánicas ${ }^{13}$, úlceras esclerodérmicas ${ }^{14}$, cierre de heridas quirúrgicas de mano $^{15}$, terapias periodontales ${ }^{16}$, regeneración de hueso dentoalveolar ${ }^{17}$, cierre de fístulas oroantrales ${ }^{18}$, cirugía de terceros molares ${ }^{19}, \mathrm{y}$ tratamiento de osteonecrosis de los maxilares asociado a antirreabsortivos óseos ${ }^{20}$, entre otros.

El objetivo de este estudio es relatar nuestra experiencia inicial en el tratamiento de heridas complejas por diversas causas con el uso de L-PRF y evaluar los resultados bajo esta técnica.

\section{Materiales y Método}

Se realizó un estudio prospectivo de una serie de casos de pacientes con diagnóstico de herida complejas de distintas etiologías que fracasaron al tratamiento de heridas tradicional, estos fueron sometidos a curaciones avanzadas con autoinjerto de L-PRF, entre enero de 2017 y diciembre de 2018, en el Servicio de Cirugía General en conjunto con la Unidad de Cirugía Maxilofacial del Hospital Santiago Oriente - Dr. Luis Tisné Brousse.

Dentro de los criterios de inclusión, se determinó que correspondía a una herida compleja si presentaba dos o más de los siguientes factores: importante pérdida de sustancia (extensión o profundidad), paciente con comorbilidades, infección local y compromiso de vitalidad y/o cronicidad.

\section{Téenica quirúrgica}

La obtención del concentrado plaquetario se realizó mediante el protocolo de centrifugado 2.700 rpm durante 12 minutos a temperatura ambiente de las muestras de sangre venosa o arterial obtenidas de cada paciente, colectados en tubos Vacutainer Serum de 6 cc. Todos los pacientes previamente se encontraban con antibiótico circulante, ya sea por tratamiento o profilaxis prequirúrgica.

En un primer tiempo operatorio, se realizó aseo 
quirúrgico de las heridas, con debridación y/o escarectomía, dependiendo del caso clínico. En el mismo tiempo quirúrgico, posteriormente se injertó el L-PRF en dos presentaciones, según la necesidad de volumen: el coágulo de fibrina sin modificación (cilindro) o en forma de membrana de aproximadamente $2 \mathrm{~mm}$ de espesor. Se instaló finalmente un apósito oclusivo que se mantuvo por 3 a 4 días, para luego descubrir la herida y realizar una curación simple de la zona. El procedimiento de L-PRF se repitió aproximadamente a los 7 días si la herida presentaba un defecto con relleno insuficiente, evaluado clínicamente tanto por profundidad como por extensión. El requerimiento de hospitalización no fue constante para todos los pacientes, ya que algunos de ellos fueron tratados ambulatoriamente.

El éxito del tratamiento se estableció por la epitelización completa de la herida, y secundariamente se evaluó la granulación completa de la misma. El fracaso fue representado por la ausencia de epitelización o granulación adecuada.

\section{Resultados}

Once pacientes con heridas complejas fueron tratados con L-PRF, 5 hombres: 6 mujeres, de edad promedio de 64 años \pm 12 , no hubo pérdida de seguimiento (Tabla 1, factores de riesgo).

El 91\% (10/11) presentó más de un factor de riesgo para la curación de la herida: $46 \%(5 / 11)$ con diabetes mellitus, $27 \%$ (3/11) fumador, 54\% (6/11) desarrollo de colonias en cultivos, $18 \%(2 / 11)$ edad mayor a 70 años, 9\% (1/11) zona irradiada previamente.

En promedio se realizaron 2,4 procedimientos por herida, con un mínimo de 1 injerto de L-PRF y un máximo de 4.

Los resultados fueron evaluados mediante el examen clínico y seguimiento de cada paciente, donde el éxito del tratamiento se consideró como la epitelización completa de la herida, que ocurrió en el $72 \%$ $(8 / 11)$ de los casos. También se midió la granulación completa de la herida, que fue del $77 \%$ (8/11), con un promedio de 7 días de evolución.

Una relación predictiva de éxito constatada fue una relación directa entre la granulación del tejido a los 7 días y la epitelización de aproximadamente $100 \%$ de la herida (Figuras 1, 2 y 3 ).

El tiempo promedio de los casos de epitelización completa fue de 41 días, con un mínimo de 30 días y un máximo de 63 días.

En relación con los 7 casos exitosos, su etiología fue variada, 2 por trauma, 4 por secuela de Infección/celulitis/fasceitis y 2 por déficit vascular (irradiación y necrosis de colgajo reposicionado). (Figuras de 3 casos)

En relación con el 27\% (3/11) de fracasos de tratamiento, los cuales no epitelizaron ni granularon adecuadamente, lo atribuimos a la presencia de un sustrato desfavorable a la técnica:

- En el primero de estos casos, en una hernia incisional gigante, se propuso potenciar el cierre por segunda intención con L-PRF, sobre una malla de vycril expuesta hace 3 semanas sin buena evolución, finalmente se le retiró la malla.

Tabla 1. Factores de riesgo de heridas compleja por paciente

\begin{tabular}{|c|c|c|c|c|c|}
\hline & $\begin{array}{l}\text { Pérdida de } \\
\text { sustancia }\end{array}$ & $\begin{array}{l}\text { Comorbilidades } \\
\text { sistémicas }\end{array}$ & $\begin{array}{c}\text { Infección/ } \\
\text { Cultivo de bacterias }\end{array}$ & $\begin{array}{c}\text { Compromiso } \\
\text { de vitalidad }\end{array}$ & Cronicidad \\
\hline 1 & + & + & + & + & - \\
\hline 3 & + & + & - & - & + \\
\hline 4 & + & + & - & - & + \\
\hline 6 & + & + & + & - & + \\
\hline 7 & + & + & + & - & + \\
\hline 8 & + & + & + & - & + \\
\hline 9 & + & + & - & - & + \\
\hline 10 & + & - & + & - & + \\
\hline
\end{tabular}




\section{ARTÍCULO ORIGINAL}

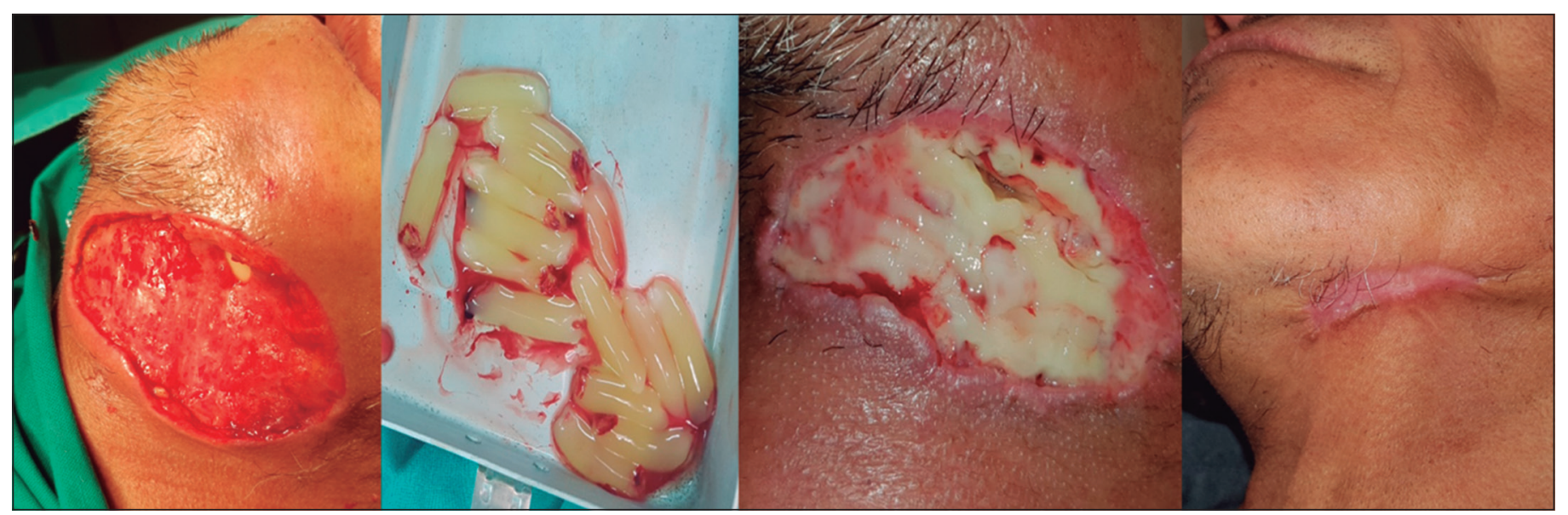

Figura 1. Caso fasceitis necrotizante, 6 semanas de tratamiento.

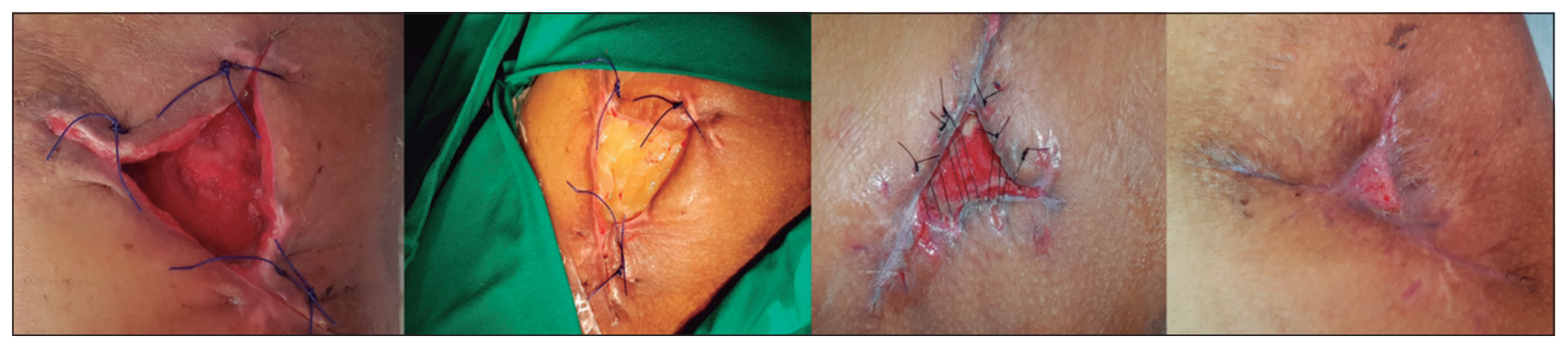

Figura 2. Caso secuela postratamiento quirúrgico de hematoma infectado en zona de muslo, 4 semanas de tratamiento.

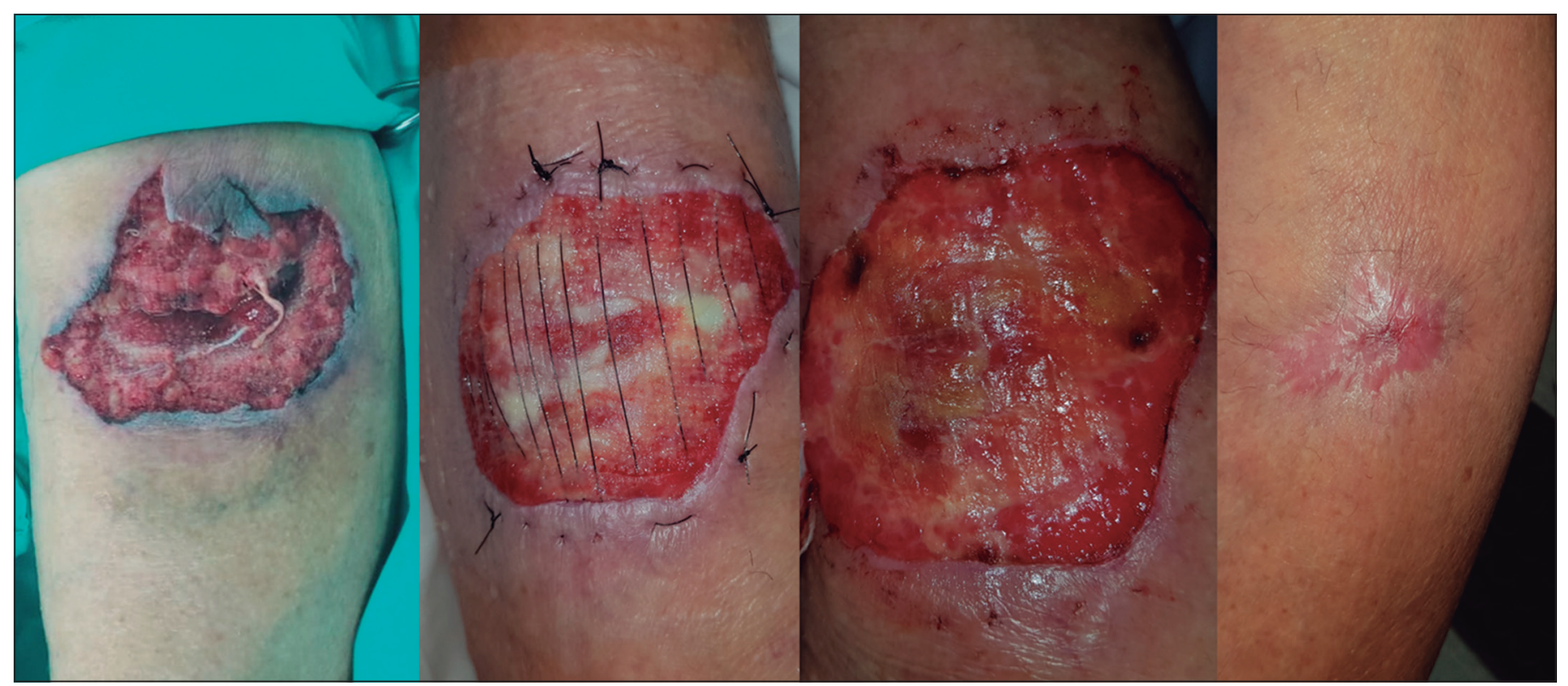

Figura 3. Caso mordedura perro en región anterior de la pierna, 9 semanas de tratamiento. 
- El segundo caso fue el tratamiento de una quemadura de tercer grado infectada en una mujer parapléjica de 71 años, que se comportaba como una úlcera por presión sacra estadio IV con una evolución de 2 meses. La dificultad de mantener el L-PRF en posición adecuada con estabilidad y contacto íntimo, generó un rápido fracaso de la técnica.

- El tercer caso fue sobre la tabla ósea externa de una tibia expuesta producto de una fasceitis necrotizante de extremidad inferior. Se realizó la fenestración de la tabla y se aplicó en el mismo acto quirúrgico el L-PRF. Creemos que el L-PRF fracasó rápidamente por la poca vascularización de la zona.

No hubo registro de reacciones adversas a la técnica. Sin embargo, en 2 casos se presentaron dificultades técnicas en el acceso a las vías venosas periféricas, que se resolvieron con un acceso por una vía arterial periférica.

\section{Discusión}

El tratamiento de las heridas complejas es variado, desde la curación tradicional y avanzada así también la utilización de otros recursos como injertos, colgajos y sustitutos dérmicos. Una alternativa adicional es el uso de L-PRF que tiene las ventajas de ser una técnica de bajo costo, de fácil obtención por su naturaleza autóloga, de manipulación simple y de pocas complicaciones. Esta técnica permite además que en condiciones ideales sea posible la continuidad del tratamiento de forma ambulatoria inmediata, disminuyendo el tiempo de estadía hospitalaria y sus costos asociados.

Por otro lado, la capacitación es breve tanto para los profesionales como para el personal de enfermería, respecto a la extracción de sangre, manipulación, y cuidados posquirúrgicos, no diferenciándose mucho de los cuidados convencionales.

Nuestra experiencia con el uso del L-PRF ha significado que mediante una intervención de bajo costo sea posible resolver heridas complejas, disminuyendo la estadía hospitalaria y/o la necesidad de tratamientos complementarios.
Consideramos que algunas condiciones indispensables para el éxito de la técnica son:

1. El acceso adecuado a la vía periférica, permitiendo el llenado del vacutainer en menos de 30 segundos.

2. Fijación del L-PRF que permita su inmovilidad en el sitio receptor.

3. El L-PRF debe estar en íntimo contacto con el tejido cruento.

4. El tejido sobre el cual se injertará el L-PRF debe tener potencial de neovascularización.

5. El sitio quirúrgico debe estar con anterioridad tratado adecuadamente (aseo quirúrgico).

Hasta la fecha, existe solo un ensayo clínico aleatorizado publicado en la literatura biomédica similar a los casos previamente descritos ${ }^{15}$. En el mismo, se evaluó el cierre por segunda intención de heridas de la palma de mano para el tratamiento quirúrgico de la enfermedad de Dupuytren con la técnica de McCash. Al comparar el grupo tratamiento, en el que se realizó una aplicación de L-PRF, con el grupo control, en que se aplicó una malla porosa impregnada con petrolato (Vasellitule), se observó un resultado favorable al uso de L-PRF, logrando la reparación en un promedio de 5 días más rápido. Comparativamente con este estudio, es muy interesante evaluar el efecto de 2 o 3 aplicaciones cada 7 días para lograr el cierre final, ya que estimamos que cada vez que se injerta con L-PRF la tasa de cierre de la herida se incrementaría.

Finalmente creemos que el tratamiento de heridas complejas con L-PRF es una alternativa factible, eficaz y segura en pacientes seleccionados

\section{Responsabilidades éticas}

Protección de personas y animales. Los autores declaran que para esta investigación no se han realizado experimentos en seres humanos ni en animales.

Confidencialidad de los datos. Los autores declaran que en este artículo no aparecen datos de pacientes.

Conflictos de interés: no hay. 


\section{Bibliografía}

1. Cronkite EP, Lozner EL, Deaver JM. Use of thrombin and fibrinogen in skin grafting. J Am Med Assoc. [Internet]. 1944 Apr 1 [cited 2019 Apr 24];124(14):976. Available from: http://jama.jamanetwork. com/article.aspx?doi=10.1001/ jama.1944.02850140022006.

2. Tidrick RT, Warner ED. Fibrin fixation of skin transplants. Surgery [Internet]. 1944 Jan 1 [cited 2019 Apr 24];15(1):90-5. Available from: https:// www.surgjournal.com/article/S00396060(44)90052-8/pdf.

3. Matras H. Die Wirkungen vershiedener Fibrinpraparate auf Kontinuitatstrennungen der Rattenhaut. Osterr Z Stomatol. 1970;67:338- 59.

4. Matras $\mathrm{H}$. The use of fibrin sealant in oral and maxillofacial surgery. J Oral Maxillofac Surg [Internet]. 1982 Oct [cited 2017 Nov 24];40(10):617-22. Available from: http://www.ncbi.nlm.nih. gov/pubmed/6981693

5. Whitman DH, Berry RL, Green DM. Platelet gel: an autologous alternative to fibrin glue with applications in oral and maxillofacial surgery. J Oral Maxillofac Surg [Internet]. 1997 Nov [cited 2017 Nov 24];55(11):1294-9. Available from: http:// www.ncbi.nlm.nih.gov/pubmed/9371122.

6. Choukroun J, Adda F, Schoeffler CVA. Une opportunite' en paro-implantologie: le PRF. Implantodontie. 2001;42:55-62.

7. Pinto NR, Ubilla M, Zamora Y, Del Río V, Dohan Ehrenfest DM, Quirynen M. Leucocyte- and platelet-rich fibrin (L-PRF) as a regenerative medicine strategy for the treatment of refractory leg ulcers: a prospective cohort study. Platelets [Internet]. 2017 Jul 20 [cited 2017 Nov 30];1-8. Available from: https:// www.tandfonline.com/doi/full/10.1080/09 537104.2017.1327654.

8. Dohan Ehrenfest DM, Pinto NR, Pereda A, Jiménez P, Corso M Del, Kang B-S, et al. The impact of the centrifuge characteristics and centrifugation protocols on the cells, growth factors, and fibrin architecture of a leukocyte- and platelet-rich fibrin (L-PRF) clot and membrane. Platelets [Internet]. 2017 Apr 24 [cited 2017 Nov 30];1-14. Available from: https://www.tandfonline.com/doi/ful 1/10.1080/09537104.2017.1293812.

9. Dohan Ehrenfest DM, Rasmusson L, Albrektsson T. Classification of platelet concentrates: from pure platelet-rich plasma (P-PRP) to leucocyte- and platelet-rich fibrin (L-PRF). Trends Biotechnol [Internet]. 2009 Mar [cited 2017 Nov 24];27(3):158-67. Available from: http://www.ncbi.nlm.nih.gov/ pubmed/19187989.

10. Kobayashi E, Flückiger L, FujiokaKobayashi M, Sawada K, Sculean A, Schaller B, et al. Comparative release of growth factors from PRP, PRF, and advanced-PRF. Clin Oral Investig [Internet]. 2016 Dec 25 [cited 2019 Apr 24];20(9):2353-60. Available from: http:// link.springer.com/10.1007/s00784-0161719-1.

11. Can ME, Dereli Can G, Cagil N, Cakmak HB, Sungu N. Urgent Therapeutic Grafting of Platelet-Rich Fibrin Membrane in Descemetocele. Cornea [Internet]. 2016 Sep [cited 2017 Nov 27];35(9):1245-9. Available from: http://www.ncbi.nlm.nih.gov/ pubmed/27362878.

12. Soufron J. Use of an autologous patch of platelet-rich fibrin (PRF) to prevent leakage after sleeve gastrectomy: A prospective observational study of 62 cases. J Visc Surg [Internet]. 2016 Aug [cited 2017 Nov 27];153(4):319-20. Available from: http://www.ncbi.nlm.nih. gov/pubmed/27282215.

13. Gür ÖE, Ensari N, Öztürk MT, Boztepe OF, Gün T, Selçuk ÖT, et al. Use of a platelet-rich fibrin membrane to repair traumatic tympanic membrane perforations: a comparative study. Acta Otolaryngol [Internet]. 2016 Oct 2 [cited 2017 Nov 27];136(10):1017-23. Available from: http://www.ncbi.nlm.nih.gov/ pubmed/27192505.

14. Shetty S, Shenoi SD. Autologous plateletrich fibrin in treatment of scleroderma ulcer. Int Wound J [Internet]. 2016 Oct [cited 2017 Nov 27];13(5):1065-6.
Available from: http://www.ncbi.nlm.nih. gov/pubmed/26290396.

15. Chignon-Sicard B, Georgiou CA, Fontas E, David S, Dumas P, Ihrai $\mathrm{T}$, et al. Efficacy of leukocyteand platelet-rich fibrin in wound healing: a randomized controlled clinical trial. Plast Reconstr Surg. [Internet]. 2012 Dec [cited 2017 Nov 30];130(6):819e-829e. Available from: http://content.wkhealth.com/linkback/ openurl?sid=WKPTLP:landingpage\& an $=00006534-201212000-00018$.

16. Verma UP, Yadav RK, Dixit M, Gupta A. Platelet-rich Fibrin: A Paradigm in Periodontal Therapy - A Systematic Review. J Int Soc Prev Community Dent [Internet]. [cited 2017 Nov 27]; 7:227-33. Available from: http://www.ncbi.nlm.nih. gov/pubmed/29026693.

17. Castro AB, Meschi N, Temmerman A, Pinto N, Lambrechts P, Teughels W, et al. Regenerative potential of leucocyte- and platelet-rich fibrin. Part B: sinus floor elevation, alveolar ridge preservation and implant therapy. A systematic review. J Clin Periodontol [Internet]. $2017 \mathrm{Feb}$ [cited 2017 Nov 27];44(2):225-34. Available from: http://www.ncbi.nlm.nih. gov/pubmed/27891638.

18. Bilginaylar K. The Use of PlateletRich Fibrin for Immediate Closure of Acute Oroantral Communications: An Alternative Approach. J Oral Maxillofac Surg [Internet]. 2017 Aug 3 [cited 2017 Nov 27]; Available from: http://www.ncbi. nlm.nih.gov/pubmed/28859924.

19. He Y, Chen J, Huang Y, Pan Q, Nie M. Local Application of Platelet-Rich Fibrin During Lower Third Molar Extraction Improves Treatment Outcomes. J Oral Maxillofac Surg [Internet]. 2017 Dec 8 [cited 2017 Nov 27]; 75:2497-506. Available from: http://www.ncbi.nlm.nih. gov/pubmed/28672135.

20. Tsai L-L, Huang Y-F, Chang Y-C. Treatment of bisphosphonate-related osteonecrosis of the jaw with platelet-rich fibrin. J Formos Med Assoc [Internet]. 2016 Jul [cited 2017 Nov 27];115:585-6. Available from: http://www.ncbi.nlm.nih. gov/pubmed/26596688. 